\title{
Study on the Application of Semiotics on the Product Design Yi Yang ${ }^{1}$ \\ ${ }^{1}$ Hubei Academy of Fine Arts, Wuhan, Hubei, 43020
}

54892713@163.com

Keywords: Semiotics; Product Design; Media

\begin{abstract}
This paper explores the application of semiotics in the field of product design. In this paper, the product is considered as a kind of integrated system and other means, and to convey the effect of performance. Essentially through an integrated product form, color, material and other visual elements of the complex technology and the inherent cultural connotation and so become an entity form, the user to complete the communication with the designer through this form.
\end{abstract}

\section{Introduction}

The last century design community the principles of semiotics applied to products in the field, that the product is a comprehensive system and other means, the performance of the role and convey language. Early in the 1920s, Scandinavian design promoters Scandinavia G package Mickelson had published "environmental symbol" theory, then, check Morse symbols Bauhaus school in Chicago to address this new century design semiotics 1950s modeling at the University of Ulm in Germany and Thomas Max Bill Maldonado et al have made a theoretical contribution. In the United States Cranbrook Academy of Art in 1983, the "product semantics Seminar" by the Industrial Designers Society of America organized by the formal introduction of the semiotic product design, and established a "product semantics" This Subject. It was since then, research Semiotics applied in product design, analysis and use of the rapid development of it.

\section{The Level of Product Symbol in "Media Related Objects"}

Information as an objective existence, there is material and non-material in two forms, the presence of substances in the form of expression for when you need a carrier, there must exist as a symbolic form to indicate the material form of information. In product design, with a certain material, structure and form of the product form it would convey full information media, it is with awareness of the designer, the user concepts are closely linked, and in different ways to convey to the user information, trying to shape its language to convey symbolic meaning and direction. In order to be considered separately from the media related matter how symbolic aspect as a medium of expression, you can open the associated objects was first ignored and interpretation related compounds. However, the content and form of the creation of course, and it should show the "what" and "for whom" linked to the performance. In general, the object is not existing as a symbolic form of the product involved in the investigation are described, but is processed into some form of media. It is associated with several aspects of form, material, color, texture and so on.

Form. Product form is the product of the information carrier, there is a coded links between it and the information is a symbol to express in material form, the designer is through the forms designed to establish channels of communication with the user. Product form is to the best advantage of various means (structural, economic, technological and mechanical) make tangible products form a clear expression of its value, meaning functions. Designers often use specific modeling language (such as segmentation and combination of physical and material selection and development and construction of innovation and utilization, etc.) product form design, the use of product-specific shape to the outside world to convey the designer's ideas and concepts. Form talk with charm lies in creating a virtual semantic space, that is, to create a user morphology the association of endless space. 
Material. With all products must have a certain quality, it occupies a space of material, in order to become material entity with a certain structure. If there is no material as a material basis, structure and function of the product there can be no existence. Achieve product form is to rely on the material to be embodied.

A material has an outer sensory. Sensory characteristics of the material is a psychological feeling, it is built on the physiological basis of human sensory organs comprehensive impression of the material, is the reaction of people feel the system due to physical stimulation of the material made by the human perception system or from the material surface information derived. Sensory characteristics of the material, also known as material texture, it is a human product by color, texture, hardness, weight, shape rough slippery feel, intuitive grasp of generating characteristics of the materials used for its quality and aesthetic feeling . Its psychological foundation is the contact person of the same material several times, due to the interaction of vision, hearing, touch and other different feelings arising synesthesia. Shape the texture of the material and products are closely linked. Important aspects of industrial products design are for certain material processing, and finally become both a physical function and mental function have products. Although the texture of the material does not change the design of the product shape, but its strong appeal, make people produce a rich psychological feelings.

Color. Not only in the form of color into the product, process structure, plays a synergistic effect, but also has aesthetic value and is very important in the visual arts as well as transfer function information. Color can not only cause people size, weight, well-being, expansion, contraction, forward and other psychological physical feeling, but can evoke a variety of emotions association, as well as emotional changes produced on this basis, the color of the symbol has sentimental value and deliver a rich cultural connotations.

Color has a face and it is possible to spread out a certain feeling that we mean, spread out the emotional response can lead moving information. Color has a strong emotional expressiveness. Contemporary American visual art psychologist Bloomer said: The color evokes emotions, express emotions, and even affects our normal psychological feelings.

\section{Product Symbol "Object Correlative" Level}

The value of symbols in that it contains certain information and expressed a certain significance. At this level, the symbol is "information bridge" between products and people, and it passes the products bearing the information to the user. The products on this level are content and that is, the product information to be passed.

As a symbol of the object, it is associated with the "characterization of the function", which is used to express an object, event or symbol outside world. In product form design, product form both expressive function, but also to explain the product quality, technological level and product quality information, etc., to express various semantic, and fully display the charm of visual symbols, the user a "persuasion". In addition to using the product features, but also has a certain mental function, meaning it expresses, and information delivered in people's mental and emotional experience, it occupies an important position. In the product design process, designers often seek exposure function and form in which something deeper, to this express special and deep meaning.

\section{Product Symbols "Explains Correlative" Level}

In the interpretation of the term here, does not refer to the interpreter or user, but to the relationship between products, product designers and product among users. Symbol of applications to achieve the purpose, is to sign interpreters (ie the product user) and sign users (that is, product designer) consistent with the meaning expressed. That is, through the products, the user is able to receive accurate product designers convey information. Symbol of communication applications with dynamic properties, the interpretation of product codes is present in the co-owned designer and user symbols reserves. First, through their own ideas, the designer the pursuit of forms of psychological feelings, after continuous concrete materialized, forming real product form. Then by 
the user will see continued solid form abstract form psychological feelings, through their past related products accumulated reserves to interpret symbols.

Disseminate Design Information. Sender (product designers) in order to make the abstract content (product information to be passed), that is the message to the recipient (product users), it must be perceived by means of symbols (products), can be prepared in accordance with the rules of a sense the message, a process called encoding or constitute [design products); sent to the recipient place through appropriate channels in the perception of the message recipient can reconstruct information based on symbolic rules, a process called translation code. Convey, the sender is the encoding process, the abstract information, by means of symbols, prepared in accordance with the rules of a symbol can be a sense of the message; the recipient is the decoding process, according to the same rules by the sensible sign letter Wen reconstruction information. We must convey elements are: sender, recipient, symbols, symbols and other rules and channel.

Condition Information Dissemination. Applications are also a cultural dissemination. In this communication process, product designers sender information, the user is the recipient of the information, products bearing the information as a product symbol, it is through this channel product dissemination. If you want to correct common provisions to achieve convey the purpose and significance constitute symbolic message, it must comply with the Recipient can understand, rather than unilateral arbitrary set by the originator. This provision is called "Code." In summary, the "code" symbols and meanings when used include convey, as well as provisions on the symbol of the binding mode. Originator reference the "code" to convey the content of the "symbolic", making the message. Message by some "lines" to the Recipient at the Recipient in reference to the "code", "interpretation" of the received message, re-constitute convey content. The specific language in the form of products regarded as a symbol, but also to make this particular product form coding, and become signifier - entity referred to, so that they can convey relevance between the designers and the public user . Sign language very strict emphasis is given meaning Code System (or letter code system) must be a coder (designer) and translation (solution) code person (user) common understanding, or it will not convey any significance. The user holds some secret aspects of the code, and user aspects to solve the problem are likely to be close to that password to what extent.

Factors Affecting the Symbol Reserves. With its potential power of entrenched influence people for the material world and the social world of perceptual evaluation criteria, particularly those inherent in the product semantics evaluation and guide people to perceive the psychological constitution. It is because the product contains the effects of perceptual concepts, which makes people's Perception added personality characteristics, different times, different objects, different countries, different social and cultural backgrounds, different lifestyles of people face the same Symbol makes products will produce different reactions, and make a different judgment and evaluation. Between different regions also have different customs, different religions, different worship idols, different cultural awareness, different values and living space. Many visual symbols is because these different semantics is not the same. As a social group generally accepted form to another group may become a new form. For example, hanging AUDI brand vehicles in Germany is mainly purchased by middle-aged character conservative customers; in the US it is yuppie's preferred means of transport; in Mexico, AUDI seen as a symbol of the height of technological development, and by progressive thinking entrepreneurs alike.

Symbol of the Convention. Symbol is a convention to determine the nature. A symbol of explanation, not only due to the direct psychological reaction caused by a vehicle symbol, and accompanied by a psychological reaction generated imagery or feelings, more importantly, there is a habit, the habit of talking about here refers to the symbol of the convention, it has a universal norm. Once created because the symbol is completed, once placed in the social field, recognized members of society, a social convention, said the significance of the mark and a social habit, a person must abide by, not free to change.

As a sign of things must be used within a certain range of people forming a convention can. If people do not know this habit, then these things will not be understood as a symbol. In other words, 
anything that relates to a symbol must be agreed upon. Only the convention can play a variety of features symbols. Design symbols can be spread meaning, interpretation and validity of spread depends on whether the recipient is capable of accurate information contained in the concept and meaning of these forms. Between the designer and the product user to understand each other, it must be based on a convention to communicate. To design a symbol for people to understand, it is that people must know in advance their agreement.

\section{Conclusion}

We can see that the spiritual life of human desire and the product has the connotation of having feelings, those dull appearance, function fuzzy, no connotation products have no market. People in the process of consumer products, the consumer no longer simply use the product features, but more consumer-oriented mental functioning, emotional interaction with the product, thus achieving the "one thing people." As a symbol of the product is the carrier of information, it is the transmission of information media. Products through its own rich and diverse forms, "language", to the user accurately deliver a large number of semantic information (such as the use of function, cultural background, symbolism, etc.), in order to achieve a pleasant exchange between persons and things.

\section{References}

[1] Lingji Yao Xu Heng Alcohol. Art and Design. Shanghai: Shanghai People's Publishing House, 2000

[2] Zhang Xianrong. Design Semiotics. Beijing: Chemical Industry Press, 2003

[3] Anquan, Li. Carmel Arthur Philippe Starck. Beijing: China Light Industry Press, 2002

[4] Qixiu Zhi, Yang Junshun. Design of Products are Based on Semiotics. Packaging Engineering, 200811.

[5] Zhang Xianrong. Design Semiotics. Beijing: Chemical Industry Press, 2004 